\title{
Using portfolio theory to assess tradeoffs between return from natural capital and social equity across space
}

\author{
Benjamin S. Halpern ${ }^{\mathrm{a}, \mathrm{b}, *}$, Crow White ${ }^{\mathrm{b}}$, Sarah E. Lester $^{\mathrm{b}}$, Christopher Costello $^{\mathrm{c}}$, Steven D. Gaines ${ }^{\mathrm{c}}$ \\ a National Center for Ecological Analysis and Synthesis, 735 State Street, Santa Barbara, CA 93101, USA \\ ${ }^{\mathrm{b}}$ Marine Science Institute, University of California, Santa Barbara, CA 93106, USA \\ ${ }^{\mathrm{c}}$ Bren School of the Environment, University of California, Santa Barbara, CA 93106, USA
}

\begin{abstract}
A B S T R A C T
Spatial variance in returns from natural resources, driven by resource dynamics and regulations, can have strong consequences for equitable delivery of value to individuals and communities. Yet resource management models implicitly weight returns equally across space, even when space is explicitly included in model dynamics and policy. Here we translate financial portfolio theory from the temporal to spatial realm and use it to quantify the inherent tradeoff between resource returns and social equity, defined as a more uniform distribution of resource value across space. We illustrate this approach with a marine case study of the Channel Islands, California, USA. Depending on the spatial distribution of resources, increasing spatial equity requires nonlinear reductions in resource returns. Realistic management options, such as effort-based fisheries regulations or marine protected areas, increase or reduce this tradeoff, respectively. We also quantify two critical advantages of portfolio approaches to management: they improve outcomes by avoiding false expectations and increase either resource return or social equity while maintaining the other.
\end{abstract}

\section{Introduction}

The idea of diversifying one's assets in order to manage risk (variability) and increase returns, i.e. building a portfolio of investments, has become widely understood and practiced. Yet it was not until the pioneering work of Markowitz that portfolio theory was developed and mathematically derived (Markowitz, 1952) and a decade or two later before portfolio approaches to asset management became commonplace. Portfolio theory has since revolutionized financial, insurance, and capital markets and has more recently been applied to diverse fields such as biodiversity conservation (e.g. Figge, 2004; Koellner and Schmitz, 2006; Tilman et al., 2006), psychology (Chandra and Shadel, 2006), computer science (Huberman et al., 1997), fisheries management (Baldursson and Magnusson, 1997; Edwards et al., 2004; Sanchirico et al., 2008; Schindler et al., 2010), and forestry (Crowe and Parker, 2008; Knoke, 2008), among others. The ecological examples demonstrate how portfolio theory can be applied to the management of natural capital, i.e. the goods and services provided by natural ecosystems. In all of these examples the fundamental guiding principle is that

* Corresponding author at: National Center for Ecological Analysis and Synthesis, 735 State Street, Santa Barbara, CA 93101, USA. Tel.: +1 805892 2531; fax: +1 805 8922510.

E-mail address: halpern@nceas.ucsb.edu (B.S. Halpern). individual assets (investment stocks, insurance policies, fish stocks, species, ecosystem services, etc.) respond uniquely over time to changes in the system, and therefore one can minimize temporal variance (i.e. risk) for a given aggregate level of return by building a portfolio of assets that respond differently (i.e. have negative covariance) to these changes.

Analogous to financial capital management, resource management generally tries to maximize the sustainable delivery of goods and services derived from natural capital (i.e. assets $=$ ecosystem services) while ensuring consistent supply of these natural resources. What is fundamentally different about resource management is that natural resources also have a spatial component of variance and covariance in their abundance and value, with resources unevenly distributed across land- or seascapes. Many resource management problems involve management measures which are spatially explicit or allocate access to resources across space, with implications for users also distributed across space. For example, spatial closures are often used to reduce fishing mortality and rebuild stocks, with the location of those closures disproportionately affecting fishermen who fished those grounds relative to fishermen who fished grounds that remain open. Although nonspatial forms of management such as uniform fisheries regulations remain common, the trend in marine resource management is towards spatially-explicit methods such as marine spatial planning.

For a large set of these management problems, there are dual objectives of (a) maximizing the total sustainable benefit to 
stakeholders of a given policy and (b) ensuring the equitable distribution of benefits to different stakeholder communities within the planning region. Often these goals are mandated by law. Even when they are not mandated, there are practical reasons for seeking both objectives - maximizing sustainable economic benefit and providing benefit to the greatest number of people has social value. In many cases efforts to maximize the overall value of natural resources favors locations with the highest value, yet if resources are patchy in space then resource users with the best access to high-value patches will benefit disproportionately from such a management approach (Blaustein, 2007; Jones, 2009). Spatial variance and covariance in resources consequently creates an important tradeoff for spatial management decisions (e.g. fishing regulations, use permits, etc.); one must balance the desire to achieve optimal overall value derived from natural resources with the need for spatial equity wherever governments and resource agencies have a moral or practical need to make access to and delivery of services more equitable among people and communities (Mutz et al., 2002).

Social inequity can lead to conflicts among individuals or stakeholder groups and stall or doom efforts to implement management reform. The need to address social equity in management decisions is widely recognized. The clearest examples occur when sets of people are differentially restricted from a resource, e.g. access to nature parks, or when groups of people are disproportionately hurt by permitted uses of areas, e.g. pollution from zoned industrial uses (Blaustein, 2007; Evans and Kantrowitz, 2002; Mascia and Claus, 2009). Indeed, compliance with management policies is often dependent on equitable access to resources, real or perceived (Sutinen and Kuperan, 1999). Here we demonstrate a novel approach to incorporating spatial evenness of ecosystem services into resource management decision-making; this approach explicitly accounts for social equity in cases where resource users are seeking an even distribution of resource returns across space. We focus on equity issues in which patchwork regulations modify access to resources within and among user groups in a spatially-explicit manner and offer quantitative analytical tools to assess these inequities. In particular, portfolio theory offers an efficient tool for proactively evaluating the tradeoffs between maximizing return from natural resources and maximizing equitability in access to those natural resources, providing critical guidance for management decisions.

The underlying premise of portfolio theory is that covariance among assets influences the variance of a collection of assets at any given level of return. This will be true when covariances are sufficiently large (relative to asset variances and returns) that they influence portfolio variance given a desired level of return. Here we focus on questions of resource management where human activities that derive value from the ocean (ecosystem services) are the 'assets', the value derived is the 'return', and where variance and covariance are measured spatially. Spatial covariance measures spatial access to assets by different users and is therefore important when management decisions affect multiple resources. As governments and agencies move towards ecosystem-based management and comprehensive spatial planning (Halpern et al., 2008; POC, 2003; USCOP, 2004), such cross-sector management will likely become much more common, as will tools necessary for addressing inherent tradeoffs. Accounting for spatial covariance allows resource managers to address issues of equity of access by groups using different resources simultaneously and provides a means to explicitly assess tradeoffs within and among ocean use sectors. Smaller spatial variance represents more even distribution of total resource value across space, and often comes at the cost of lower portfolio returns.

To measure the value added by a portfolio approach, its performance needs to be compared with solutions that do not use, or are unaware of, covariance. That is, comparisons need to be made to traditional, or analyst, approaches where covariance among natural resources is thought to not exist ('naïve analyst') or is known but ignored ('informed analyst'). This evaluation framework leads to two types of comparisons: (1) how does the 'naïve analyst' expect to perform relative to the actual results that occur given consideration of covariances among natural resources (portfolio approach); and (2) how does the portfolio approach perform relative to the 'informed analyst' where covariances are ignored when allocating access to natural resources? The former comparison addresses false expectations that arise when real consequences of covariance are not accounted for, while the latter comparison addresses the true value added of a portfolio approach relative to the analyst approach. Theoretically, the sign and magnitude of the covariance values influence whether the naïve analyst's expectations exceed or fall short of the informed analyst result (Supplementary Fig. 1). When covariance values are relatively large (positive or negative), portfolio solutions are expected to strongly outperform the informed analyst, because the portfolio 'investor' knows to avoid assets (natural resources, or specific locations) that covary strongly and positively and to invest in assets that covary strongly and negatively (Supplementary Fig. 1). That is, consideration of covariances helps reduce portfolio variance and achieve better returns. The magnitude of returns and variances for individual natural resources, relative to the magnitude of covariance, also will affect the degree to which the portfolio solution outperforms the informed analyst (Sanchirico et al., 2008).

We make three important contributions in this paper. First, we demonstrate the application of portfolio theory to resource management questions where spatial variance in natural capital is important. Ultimately natural resource management must consider both spatial and temporal variance simultaneously, but for clarity we focus solely on spatial variance here. We illustrate the translation of portfolio theory to spatial variance using a marine case study based on valuation estimates of several marine resources around the Channel Islands, California, USA. These estimates include two sets of ecosystem services measured in very different units, which show how portfolio theory can be applied to the spatial management of a diversity of resource types. Second, we use the case study to address several questions aimed at understanding the consequences of asset covariance that are important for the application of portfolio theory within ecosystem-based management: (1) what is the nature of the tradeoff between portfolio return and social equity, when social equity is defined by the spatial evenness in access to natural resources? (2) what are the costs of failing to account for covariance on management expectations (the naïve vs. informed analyst comparison) and on actual management outcomes (informed analyst vs. portfolio investor comparison)? and (3) how does portfolio analysis change optimal asset allocation relative to a non-portfolio (analyst) approach? Our third contribution is to explore how portfolio theory can guide establishment of spatially-explicit management and zoning plans, including fishery closure zones. We demonstrate how portfolio analyses can be used to compare management options and, importantly, how the theory can generate spatially-explicit management recommendations. We also discuss where and when management would likely benefit from the application of portfolio theory and highlight key remaining research gaps that need to be filled before portfolio management could be fully operationalized.

\section{Methods}

Portfolio approaches to asset management assess the tradeoff between expected risk (variance) and return, where return on the portfolio $\left(\mu_{P}\right)$ is the sum of total available returns on each asset 
$\left(\mu_{j}\right)$ times its proportion in the portfolio $\left(X_{j}\right)$ across the full range of possible asset allocation schemes $(N)$ in the portfolio:

$\mu_{P}=\sum_{j=1}^{N} X_{j} \mu_{j}$

and portfolio variance $\left(\sigma_{P}^{2}\right)$ is the sum of summed proportional variances and covariances:

$\sigma_{P}^{2}=\sum_{j=1}^{N} X_{j}^{2} \sigma_{j}^{2}+\sum_{j=1}^{N} \sum_{\substack{k=1 \\ j \neq k}}^{N} X_{j} X_{k} \operatorname{COV}_{j k}$

Portfolios can be compared based on their return relative to their variance, with an 'efficiency frontier' emerging where the ratio is optimized given constraints in the management scenario (e.g. use of no-take zones) and asset data (see below for derivation of the efficiency frontier). Portfolios with ratios that lie interior to the efficiency frontier are always sub-optimal decisions, regardless of one's tolerance of risk, since one could increase the return at no change in risk or maintain the same return while reducing risk. Along the frontier, portfolio selection is based on the desired balance between return and risk.

We focused on a case study already subject to extensive research and management efforts, the Channel Islands National Marine Sanctuary (CINMS), California, USA. We used economic revenue data for 14 commercial fisheries (measured in dollars) and human use data for seven consumptive and non-consumptive recreational activities (measured in person-days) within the CINMS that were collected for the process to establish a network of marine reserves around the islands in 2003 (Leeworthy et al., 2005). The fisheries include wetfish, squid, kelp, urchins, lobster, sheephead, flatfishes, sea cucumber, seabass, sharks, crabs, prawns, shellfish, and tuna; and the recreational activities include charter/party boat fishing, charter/party boat consumptive diving, private boat fishing, private boat consumptive diving, recreational diving, whale watching, kayaking, and sailing. Collectively these resources are termed 'assets' here, following the terminology of portfolio theory. Two very small marine reserves had existed around one of the islands, Anacapa Island, during this time period that likely had negligible effect on spatial variance of fisheries or recreation returns; all other management regulations were uniform across the planning area. Recreation data were collected in 1999, except charter/party boat data, which were collected in 2003. Fishery data are an average from landings reported from 1996 to 2003 or from 1996 to 1999, depending on the fishery. More details on data collection methods can be found elsewhere (Leeworthy et al., 2005). Values are reported for $1^{\prime}$ blocks (roughly $1.5 \times 1.5 \mathrm{~km}$ ) totaling 3000 blocks surrounding the islands (roughly $6700 \mathrm{~km}^{2}$ total). These reporting blocks were used as the planning units for our spatially-explicit analyses, described below. We analyzed the two classes of assets separately to avoid the challenge of comparing assets measured in different units, and focus most of our analyses on the fisheries data for illustrative purposes. Roughly half the planning area had non-zero values for at least one fishery (1736 blocks) or recreational (1626 blocks) service (Supplementary Fig. 2); for each type of asset (fishery or recreational) our analyses focus on this subset of planning units. Delineation of larger areas containing zerovalue planning units is arbitrary and inclusion of these units would artificially affect variance and covariance of assets. We assume these historic values represent sustainable levels of harvest.

Using basic portfolio theory described above we calculated returns and variances for 10,000 simulated portfolios. Each portfolio's 'investment' strategy was determined by random assignment of the proportion of the total available value of each asset (i.e. fishery or recreational activity) to be included in the portfolio $\left(X_{j} \in[0,1]\right.$ for each asset $\left.j\right)$ across the entire planning region, without indicating specific values at each particular planning unit. Although in practice each portfolio would require specific spatial management, we do not explicitly define specific asset values at each planning unit because the number of combinations is too large to explore numerically (except when $X_{j}$ is zero or one), and only $X_{j}$ is needed for calculating the portfolio's return and expected variance (via Eqs. (1) and (2)). Thus, implicitly this analysis considers each $1^{\prime \prime}$ block to potentially have any proportional amount of the value of each asset 'delivered' from that block, and the portfolios represent theoretically possible but realistically difficult management solutions that would set a unique harvest or access value for each asset for each block. This is analogous to financial portfolio analysis that assesses a full range of investment strategies for any possible level of investment across assets and over time.

For each simulated portfolio, return was calculated as the sum of the total potential value of the natural resource across the whole planning region multiplied by its randomly assigned proportion in the investment, and expected spatial variance was determined in relation to proportional investment levels for each resource and the spatial variances and covariances of the resources (Eqs. (1) and (2)). We computed the portfolio efficiency frontier of returns and variances, which represents the set of optimal solutions, and associated resource proportions by maximizing a multi-criterion objective function that simultaneously accommodates return and variance,

$O b j=\max \left[\alpha \mu_{p}-(1-\alpha) \sigma_{p}^{2}\right] \leftarrow$

where the coefficient $\alpha \in[0,1]$ controls the relative weight of return vs. variance in the overall objective. Multi-criterion objective functions are commonly used when the goal is to optimize more than a single function (Ehrgott, 2005). When $\alpha=1$ return is maximized without regard to variance, whereas when $\alpha=0$ variance is minimized. Portfolio and naïve analyst efficiency frontiers were calculated with and without consideration of covariance values (Eq. (2)), respectively. Informed analyst efficiency frontier variance values were calculated using asset proportions, $X_{j}$, associated with the naïve analyst's efficiency frontier but with asset covariances included in the calculation of portfolio variance. Informed and naïve analyst solutions were therefore identical in portfolio returns but not variances for any set of asset proportions.

For the fishery category we also explored more realistic management scenarios that regulated resource extraction effort levels nonspatially (affecting the proportion of each species' value that is accessed by fisheries) or included the use of spatially-explicit no-take (no-access) closures. For the non-spatial fisheries regulation scenario, we considered two approaches: setting spatially uniform effort levels that are equivalent across all fisheries, and setting spatially uniform effort levels specifically for each species. In these analyses, portfolio return is calculated with the constraint that $X_{j}$ is equivalent across all patches. With uniform effort equivalent across all species we were able to explore the full range of effort levels $(0-1.0)$, the outcomes of which delineate the efficiency frontier. For species-specific effort we considered outcomes from 10,000 different combinations of spatially uniform effort levels among the species, then identified the collection of outcomes (i.e. management strategies) that delineate the efficiency frontier of optimal return:variance ratio. We did not explore solutions that kept overall return for each asset the same while modifying the contribution to this whole from each block as these solutions would require increasing the return (in some cases dramatically so) from individual blocks, which is not ecologically possible in many cases.

For reserve closures, we set the value of certain blocks to zero and varied the number and location of these blocks randomly to explore possible management scenarios. 
As with the asset-specific, effort-based management scenario above, we evaluated outcomes and delineated the efficiency frontier under management with reserves using numerical simulations. We iteratively considered $n=0,1,2,3, \ldots, N$ planning units to be closed to fishing (i.e. in reserves) and, for each iteration, simulated 10,000 random distributions of reserves across positive-value planning units in the domain $(N=1736)$. For each simulation we calculated: (1) cumulative value of landings available to the fishery across all species and non-reserve planning units, $\mu_{\text {p.reserve }}$, (2) proportion of each fishery's total return that is in non-reserve areas,

$X_{j, \text { reserve }}=\frac{\mu_{p, j, \text { reserve }}}{\mu_{p, j}}$,

and (3) portfolio variance, $\sigma_{P}^{2}$, in relation to $X_{j \text {,reserve }}$ and the spatial variances and covariances of the resources. In these analyses $X_{j, \text { reserve }}$ is implicitly spatial based on the spatially-explicit siting of reserves across the domain. This approach enables us to remain within the original portfolio theory framework and maintain a fair comparison across the management approaches evaluated in this study. We traced the outer edge of return and variance levels produced by the full set of simulations to identify the spatial portfolio efficiency frontier (Supplementary Fig. 3). Closing all units to fishing (100\% marine reserve coverage) dictates $X_{j}=0$ for all $j$ and a resulting portfolio with zero return and variance; not closing any units to fishing (no reserves) allows $X_{j}=1$ for all $j$ and portfolio return and variance are maximized. Consequently, portfolio efficiency frontiers for solutions that use partial fishing regulations within each planning unit and those that use no-take reserves meet at their extreme ends. A sub-set of no-take reserve portfolio simulations, representing $20 \%, 40 \%, 60 \%$ and $80 \%$ of the positive-value planning units in reserves ( $n=347,694,1042,1389$, respectively), were plotted to present a specific set of solutions at, and interior to, the frontier solutions.

To evaluate how spatial portfolios actually look on the seascape, we mapped a sub-set of possible solutions of portfolio results across a range of portfolio return values. We mapped two sets of no-take reserve solutions: those from the unconstrained no-take efficiency frontier (arbitrarily chosen at 14\%, 25\%, and 37\% setaside to span a range of values that are often achieved or aimed for in MPA networks) and those from a constrained no-take efficiency frontier (fixed percent set-aside). For the constrained solutions, we focused on $25 \%$ no-take set-aside to represent a common target set-aside for current no-take reserve planning efforts.

Comparison of portfolios can be guided by a risk-adjusted performance index such as the Sharpe Index (Sharpe, 1966), which quantifies the ratio of returns to variability (standard deviation):

$\theta=\frac{\mu_{p}-R_{f}}{\sigma_{p}}$,

where $R_{f}=$ risk-free rate of return and is set to zero here. The Sharpe Index is a common financial measure that standardizes expected return by expected risk along efficiency frontiers to help highlight differences among management options. We calculated Sharpe Index values from management outcomes along the optimal portfolio and naïve and informed analyst efficiency frontier solutions to highlight their differences in value.

In the context of this study, social equity in natural resource management is achieved by minimizing spatial variance in the distribution of asset returns across the planning region. This goal assumes a uniform distribution of resource users across the region; in reality users are spatially concentrated and thus have varying abilities to access different patches (regardless of a patch's potential value once accessed). To account for non-uniform user distribution we re-ran our analyses after modifying the original CINMS revenue data for each patch by the average proportion of revenue spent (i.e. the cost) of accessing that patch by users concentrated in the three main fishing harbors in the Santa Barbara Channel region (Santa Barbara, Ventura and Oxnard; Supplementary Fig. 2). For each harbor we assumed the value of the species within a patch (species revenue - cost of access) to decrease exponentially with increasing distance between the user and the patch; we explored multiple exponential relationships of varying strengths (see Supplementary material for equations). We then averaged the values among the three harbors, and used this mean value of each asset in each patch in our analyses. Thus, under this modified analysis, social equity is achieved by minimizing spatial variance in the distribution of realized asset returns (value minus cost) to users located in Santa Barbara, Ventura and Oxnard harbors.

\section{Results and discussion}

\subsection{Tradeoff between return and social equity}

Two key results emerge from applying portfolio theory to spatial management questions. First, optimized tradeoffs between the value of natural resources and social equity in the spatial access to those resources (i.e. the efficiency frontier) are concave (Fig. 1A; Supplementary Figs. 4 and 5), as predicted by theory (Markowitz, 1959). In other words, initial increases in return (i.e. fisheries value) can be gained with relatively little cost to social equity goals, which is measured by portfolio variance, but further increases in return require proportionally greater spatial inequity. This tradeoff between return and equity can be represented as either the total or marginal cost to portfolio return of greater social equity (Fig. 1E). The marginal cost curve shows the decrease in return for each incremental increase in social equity, and in our case study the final increments in equity come at a great cost to returns. Total cost is the maximum return on the efficiency frontier minus the return on the frontier associated with a particular variance. It allows one to compare the absolute change in return between any two levels of social equity, and shows how moderate to high levels of equity require significant reductions in portfolio return. Both methods of measuring cost illustrate why otherwise high-return (e.g. profitable) resource management decisions can often face significant stakeholder resistance because they are associated with high levels of inequity.

These analyses highlight a range of solutions; the preferred location along the frontier will depend on social and political preferences within the management jurisdiction and the possibilities and capabilities for offsetting undesirable increases in social inequity. Preferences will vary from region to region and can be represented by "indifference curves." Where an indifference curve is tangent to the frontier, the optimal tradeoff between equity and portfolio resource return is achieved for a given community (discussed further below). Not all solutions are equally achievable because the ability of resource users to adapt to new regulations can vary within communities. One must know the efficiency frontier, societal preferences for spatial equity vs. overall resource return, and the feasibility of options to find a socially-optimal management solution. Portfolio analysis provides the frontier solutions, economic surveys can elucidate societal preferences for risk vs. return to determine indifference curves, and policy assessments can identify which management solutions are feasible.

A second key result is that many management options lead to outcomes that fall below the frontier. These are clearly inefficient since other options exist that could increase both social equity and overall returns through strategic management that modifies harvest or access in different blocks according to the spatial covariance of the resources. Such an approach is being proposed as part 

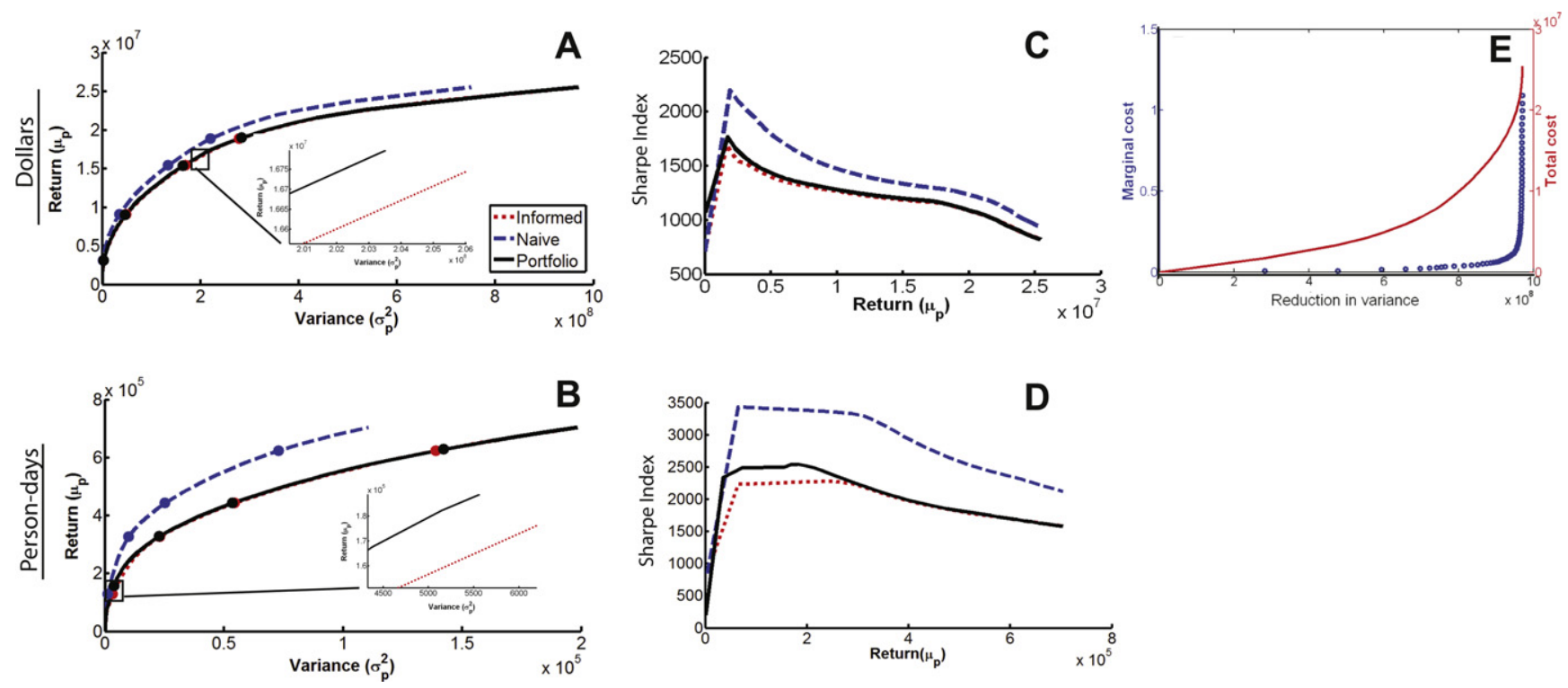

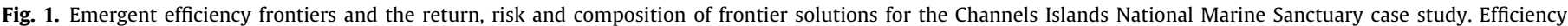

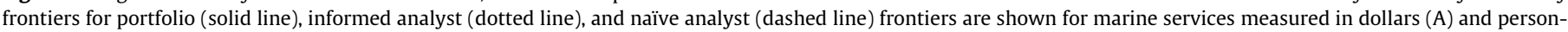

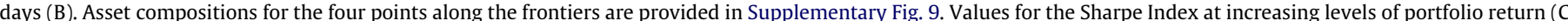

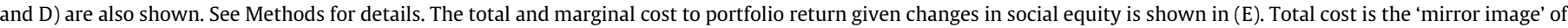
the efficiency frontier in (A). Insets in A and B show enlarged regions of the efficiency frontiers to show areas of greatest divergence between the two frontiers.

of marine spatial planning in countries around the world, including the US Interagency Ocean Policy Taskforce recommendations (CEQ 2010). Delineating the efficiency frontier enables one to find such win-win solutions to replace competing management options that are perceived to have tradeoffs but are actually sub-optimal choices, i.e. below the frontier. Both results have been shown repeatedly in past portfolio analyses but are important to highlight in the context of spatial resource management.

\subsection{The consequences of resource covariance}

When the distribution of natural resources is patchy, management faces an inherent tradeoff between social equity and overall returns. Portfolio theory provides a method for identifying optimal solutions and assessing tradeoffs among policies. Indeed, false expectations from naïve analyst solutions are significantly higher than what is likely possible given innate spatial covariance of the resources (Fig. 1A and B). In particular, the naïve analyst expects a given return with much less risk, or higher social equity, than is possible. Such false expectations may be particularly problematic when adjusting management strategies is neither trivial nor rapid, and management failure has significant political, social, and environmental costs.

In contrast, portfolio solutions always out-perform informed analyst solutions because analyst solutions do not account for real covariance and the added value of a portfolio approach is always positive. The differences in return are relatively small in this case study, primarily because the covariances among natural resources are predominantly positive and variances of individual resources are quite large (Fig. 1A and B; Supplementary Tables 1 and 2). Even so, portfolio solutions still out-perform informed analyst solutions by up to 5\% (Supplementary Fig. 6). This result highlights the ability of portfolio management to increase overall returns at no added cost to social equity (i.e. change in variance).

Subtle differences between portfolio and informed analyst solutions in our case study are more clearly illustrated after standardizing expected return with respect to expected risk (portfolio variance) using the Sharpe Index (portfolio return divided by its standard deviation, with risk free return set to zero; Fig. $1 \mathrm{C}$ and D). This type of standardization focuses attention on the amount of extra return that is gained with each increase unit of risk (Sharpe, 1966), or in the context of this study, incremental decrease in social equity. Larger differences in this index are primarily at low to medium levels of portfolio return, which is expected because at these levels variance can be minimized (i.e. social equity maintained) through strategic investment of small amounts of resources that have high returns and low spatial covariances. In contrast, large returns can only be achieved by including nearly all of each resource, in which case decisions are less strategic in relation to covariances and instead guided predominantly by returns.

Differences between the performance of portfolio and informed analyst approaches are expected to be more pronounced at higher return levels when assets (e.g. ecosystem services) cannot co-occur. Since they covary strongly and negatively, higher returns in one asset come at the cost of much lower returns in another asset. This result was not observed here because the assets in our case study had relatively small and primarily positive covariances. We expect negative spatial covariance to be more prevalent for assets tied to more diverse types of natural resources, rather than the fisheries-only and recreation-only examples analyzed here. For example, water filtration provided by estuarine habitats will have a very different spatial pattern than fisheries production (which tends to be concentrated on continental shelves), leading to negative spatial covariance between assets derived from the two.

It is also instructive to compare solutions derived from a portfolio approach to those under different management scenarios that may be more feasible to implement. We compared portfolio solutions to (1) networks of no-take reserves (of varying locations and sizes) where all fishing (or other types of activities) is excluded and (2) two forms of traditional effort-based, non-spatial regulations (without reserves). Optimal no-take reserve solutions performed significantly better than solutions with uniform fishing effort (across all assets) because spatial solutions allowed 


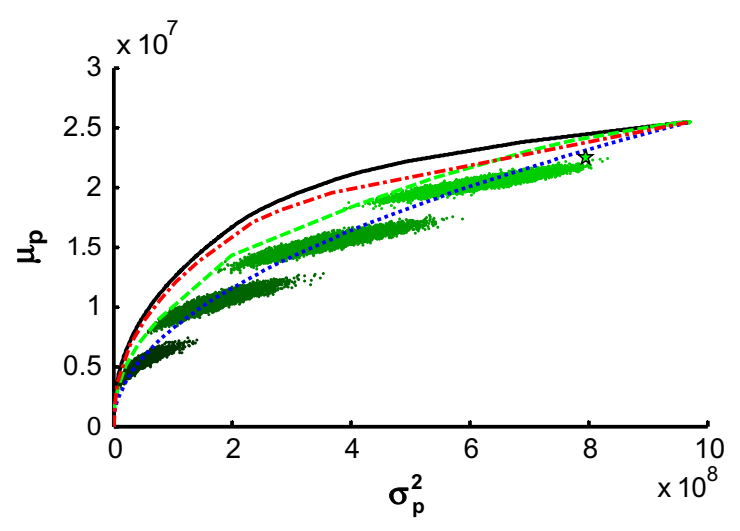

Fig. 2. Efficiency frontiers (lines) and simulated no-take reserve portfolios (points) for different management scenarios. Scenarios include optimal portfolio (solid line), regulation of effort levels specifically for each species (dash-dot line), use of no-take areas (dashed line), regulation of uniform effort levels across all assets (dotted line), and simulated no-take solutions at increasing fixed percentages of no-take area (increasing darkness of gray points: $20 \%, 40 \%, 60 \%$, and $80 \%$ respectively). The star (upper right) represents the actual MPA network implemented in 2003 with 19\% no-take areas.

managers to strategically select locations with variance and covariance structure that optimized the portfolio's return:variance ratio (Fig. 2), for example, areas with high return and low variance and/or negative covariance. Essentially, one can maintain overall return on natural capital while increasing social equity by more carefully selecting where resources can be extracted, an approach that is not possible through effort-based regulations. In contrast, optimal no-take reserve solutions performed significantly worse than optimal asset-specific, non-spatial fishing effort regulations. Here, the latter policy allows for fine tuning of effort levels for each species, which improved the return:variance ratio relative to the more blunt all-or-no effort spatial options under reserve management. In other words, one can improve portfolio performance by more carefully selecting the proportion of each resource to be extracted, an approach that is not possible solely through creation of no-take reserves. It follows logically that all of the more feasible management scenarios that we evaluated performed worse than the best, but impractical, policy that allocated the optimal (and unique) proportion of each resource to be extracted from each patch in the planning region.

Simulations of four different levels of no-take reserve protection (closing $20 \%, 40 \%, 60 \%$, or $80 \%$ of fished areas) show two additional key results (Fig. 2). First, about half of all no-take reserve solutions outperform uniform effort regulations, suggesting a positive role for no-take reserves in marine spatial planning. This is consistent with other results simulating the performance of no-take reserve networks relative to non-spatial management (Airame et al., 2003). Second, each cloud of points in Fig. 2 ranged substantially along the $x$-axis and minimally along the $y$-axis, indicating that one can achieve roughly similar returns at a given percent of area set aside in reserves using a wide range of possible reserve solutions, but with dramatically different levels of social equity. For example, when $40 \%$ of the planning area is set aside in no-take reserves (medium gray points in Fig. 2), optimal management plans yield roughly $\$ 15$ million in return, but social equity across possible solutions differs as much as three-fold. Consequently, careful selection of reserve locations can significantly improve social equity at little cost to portfolio return.

In 2003 a network of marine reserves was implemented around the Channel Islands (star in Fig. 2). This real-world solution is relatively close to the portfolio frontier, which is encouraging but also not surprising because economic factors and spatial concerns played an important role in the reserve design process (Airame et al., 2003). As noted above, this encouraging result is consistent with theoretical expectations that the return is very high where analyst and portfolio solutions converge. Furthermore, the realworld solution sits nearly perfectly atop the $20 \%$ no-take efficiency frontier (light gray points in Fig. 2), indicating that the return:variance ratio of the final solution was close to optimal. However, the real-world solution was interior to the optimal, unconstrained notake portfolio efficiency frontier that considers all possible amounts of set aside. As a result, the high returns provided by the real-world solution came with a higher risk (greater social inequity) than was possible with theoretical optimal choices. Moving left from the star to the no-take efficiency frontier in Fig. 2 illustrates how the same return could have been achieved at much lower portfolio variance. That is, a portfolio approach to reserve selection could have produced solutions that provided even higher return with greater social equity - an economic win-win situation.

\subsection{Mapping portfolio solutions}

To be most useful to management efforts, portfolio solutions need to be translated into spatially-explicit management options. This is not a trivial task as the modeled optimal portfolio solutions apply unique regulations (e.g. allowable catch) for each resource in every single planning unit, which is practically infeasible to implement and enforce. To translate the general results into spatially-explicit maps, we focused on the no-take reserve solutions. We examined representative points on the unconstrained (i.e. any \% set aside) and 25\% no-take reserve efficiency frontiers and found several interesting results (Fig. 3). First, optimal solutions are scattered and not clustered into zones that would be easy to manage and enforce. Including management cost may render these infeasible, even if they are optimal from a social equity perspective. Clustered solutions such as the actual network of reserves established around the islands can produce results near the efficiency frontier (Fig. 2), but this result has higher social inequity (i.e. high portfolio variance) than is theoretically achievable with optimal spatial regulations. Management constraints such as enforceability or fishers' preferences for different types of management (e.g. McClanahan et al., 2008) may therefore severely constrain the ability to achieve social equity because optimal management plans may not be feasible. However, using planning tools such as MARXAN that can search for solutions that optimize return against costs could be used in tandem with our portfolio approaches to find solutions clumped in space. Second, the specific locations and pattern of reserve closures change significantly as one moves along either frontier, suggesting that increasing the desired return on a portfolio is not simply a matter of adding area to (or even preserving) existing closures.

Importantly, our approach to analyzing and mapping spatial portfolio solutions assumes that social equity increases linearly with increased evenness in the distribution of asset value across the management. Even distribution of return across space should approximate reality at very large and very small spatial scales. In the former case, many communities along a large coastline are accessing different patches of the ocean for economic value. In the latter case, many people have easy access to all the same locations. However, this assumption may not hold when human populations or access points for resource stakeholders (such as fishing ports) are unevenly distributed. In these cases, social equity would be defined heterogeneously across space, and thus would correspond nonlinearly with changes in spatial variance and covariance. To assess the implications of this scenario we modified the value of the assets in each patch in relation to the cost of accessing the patch by users centralized in three harbors in the planning region (Supplementary Fig. 7). Given this modification, social equity 


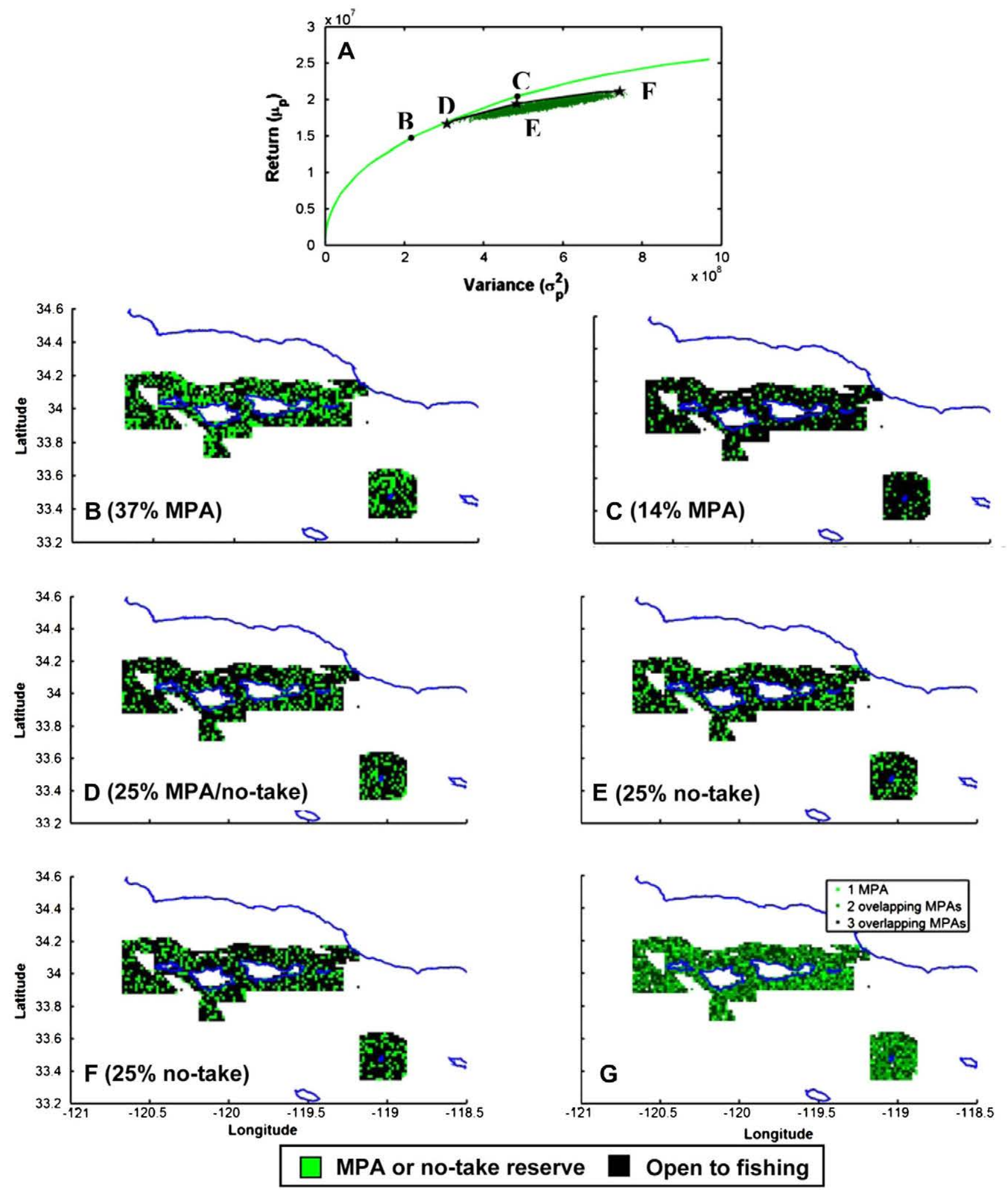

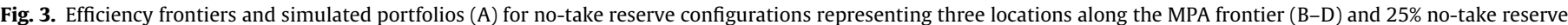

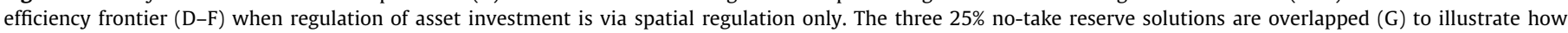

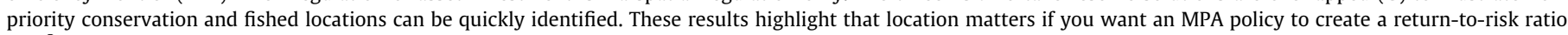
$\left(\mu: \sigma^{2}\right)$ near the true efficiency frontier.

represented a distance-weighted spatial variance in the distribution of asset returns to users in Santa Barbara, Ventura and Oxnard population centers. Although more realistic, consideration of social equity in relation to spatially heterogeneous levels of resource va- lue among user communities did not change the qualitative interpretation of our results (Supplementary Fig. 8). Most importantly, the relative performance of the optimal portfolios (i.e. the efficiency frontiers) for the different management scenarios evaluated 
did not change (Supplementary Fig. 8). Furthermore, no-take reserve locations associated with points along the no-take reserve efficiency frontier did not change conspicuously.

\subsection{Practical concerns for spatial portfolio management}

With variance measured spatially, rather than temporally, the scales of both the input data (i.e. spatial resolution) and the management planning area (i.e. geographic scope) are critical. For small planning areas or course input data, spatial variance and covariance of assets will be greatly influenced by the number of planning units, primarily because variance stabilizes as sample size increases. At very large planning scales, or for management regions that span oceanographic or biogeographic boundaries, variance will be naturally higher - this is also the scale at which social equity concerns begin to play a more important role (for example, the equity in catch landed among ports can dramatically affect the viability of a spatial management plan). The relatively small scale of our case study masks the fact that it spans a major biogeographic boundary, which may create large spatial variance between the biogeographic regions and large (incidentally positive) spatial covariance among fisheries. Together, these traits contribute to the stronger similarity between portfolio and analyst results in our analyses. Analyses within separate biogeographic regions would likely lead to much larger benefits from the portfolio approach.

Access rights to resources, understood broadly here as access to the value of a location for certain uses of the ocean rather than for particular individuals, is an increasingly common approach to resource management, in particular through zoning regulations and marine spatial planning. We have shown here that incorporating the spatial variance in the value of resources into management plans can improve their overall value and/or the equitable distribution of that value to stakeholders, but we recognize that efficiency (and effectiveness) in management is also due to many other factors, including ease of enforcement, philosophical views of whether it is appropriate to limit access to resources to certain groups of people, or cheaper (if not as effective) non-spatial reforms (such as fishing gear regulations).

In our translation of porfolio theory from the temporal to the spatial realm we used a standard financial portfolio theory framework that estimates returns and variances based on an investment strategy of assets and their temporal values and covariances. Accordingly, we assessed the performance of investment strategies with respect to the distribution of assets and their spatial covariances. To remain within the original portfolio theory framework spatial management plans (e.g. reserves) were converted to spatially implicit asset investment strategies $\left(X_{j}\right)$. This approach helped maintain a robust qualitative and fair quantitative comparison across the different spatial and non-spatial management approaches evaluated in our study, however it leaves room for assessing how expanding the financial portfolio theory framework to include explicitly spatial investment decisions affects the tradeoff between return from natural capital and social equity across space. In particular, we expect exploration of this step to guide the actual design of particular management policies (e.g. marine reserves).

Translation of portfolio theory from stocks and assets in financial markets to managing ecosystem services, or natural capital, provides important insights for management; yet it also poses a key practical challenge. Many ecosystem services do not have existing markets or simple methods for valuing a given unit of service (e.g. spiritual value of protected species), whereas portfolio theory has been developed for assets in a common unit of measure (e.g. dollars). One means for comparing the value of services is to estimate an indifference curve, a representation of bundles of ser- vices for which one has equal preference (Varian, 2005). For example, if society had equal preferences for Bundle $1\left(X_{1}\right.$ level of fishing and $Y_{1}$ level of kayaking) and Bundle $2\left(X_{2}\right.$ level of fishing and $Y_{2}$ level of kayaking), those two bundles would lie on the same indifference curve. Any given curve corresponds to a unique total value or utility, but all points on a curve are "equal" from the perspective of individual or societal value. Development of these curves requires one to assess relative value of assets, but not necessarily to convert that value into dollars. Indifference curves are downsloping and typically convex, because per-unit value of goods or services generally increases as that good or service becomes scarcer. As a result, indifference curves are not a simple linear relationship for which one could scale two services to the same units. Estimating indifference curves requires knowledge about an individual's or society's willingness to pay or willingness to accept different services at the expense of others (Gatto and de Leo, 2000).

In our analysis there were several other assumptions that deviate from reality but were necessary for this initial conceptualization of the problem. For example, we assume that each unitvalue for each sector is equivalent (e.g. one person-day of whale watching equals one person-day of sailing), that there are no interactions among assets beyond their covariances, and that variance and covariance matrices are static and defined. For ecosystem services that are heavily influenced or determined by biological processes (which is true for most services), this latter assumption of independence is unlikely to hold true. For example, biophysical processes that drive changes in primary productivity can create different states of service delivery (e.g., El Niño vs. La Niña conditions), and changes in predator or prey abundances due to management decisions will likely cascade through the food web and thus alter covariance structure in response to portfolio choice, in turn affecting the temporal variance of each individual resource. The financial world is rich with methods for dealing with temporally dynamic variance and covariance in portfolios, such as autoregressive conditional heteroscedasticity $(\mathrm{ARCH})$ and related models (Bollersley, 1986; Engle, 1982). Translation of this theory into the combined spatial-temporal realm is an important future research avenue.

\subsection{Conclusions}

The application of portfolio theory to natural resource management provides a powerful and flexible tool for allocating human uses spatially in a way that optimizes the tradeoff between return on natural capital and equitable spatial returns from resource use. In particular this approach allows one to identify a collection of optimal management options and thus highlights the vast majority of policies that are inferior. Portfolios are derived from assessments of spatial variance and covariance but do not necessarily prescribe the exact spatial allocation of those uses, only the proportion of the portfolio that is comprised by each use. Decisions about how to achieve those proportions are represented by constrained, but more feasible management scenarios. In our case study, we explored various constrained scenarios and illustrated one method for identifying potential priority conservation (no-take reserves) and fished areas (overlap solutions, Fig. 3G). Additional constraints are clearly possible, such as choosing core reserve locations and then building larger zones around those locations (i.e. a criterion of adjacency), but may come with significant costs to social equity. Furthermore, some allocation criteria may produce solutions that are sub-optimal, providing a useful check on the costs or benefits of different decision rules relative to portfolio management.

In general, efforts to optimize the spatial allocation of human activities have focused on single assets or sets of very similar assets or have used an additive approach that ignores service covariance. Such service-by-service approaches are clearly necessary in 
moving towards more comprehensive management, yet portfolio theory suggests they will often be sub-optimal to formal portfolio approaches, particularly in terms of achieving equitable distribution of a suite of services across space. As more data on the spatial delivery of marine ecosystem services and their valuation become available, portfolio analyses will hopefully become more common place. The recent interest in ecosystem-based management and marine spatial planning (e.g. ocean zoning) at various local, national, and international levels presents an important opportunity to apply portfolio solutions to management plans.

\section{Acknowledgements}

This work was produced as part of the Sustainable Fisheries Group, funded by the Paul G. Allen Family Foundation. Thanks to Peter Wiley and Bob Leeworthy for help in acquiring valuation data for services around the Channel Islands. All data are available upon request.

\section{Appendix A. Supplementary material}

Supplementary data associated with this article can be found, in the online version, at doi:10.1016/j.biocon.2011.01.019.

\section{References}

Airame, S., Dugan, J.E., Lafferty, K.D., Leslie, H., McArdle, D.A., Warner, R.R., 2003 Applying ecological criteria to marine reserve design: a case study from the California Channel Islands. Ecological Applications 13, S170-S184.

Baldursson, F.M., Magnusson, G., 1997. Portfolio fishing. Scandinavian Journal of Economics 99, 389-403.

Blaustein, R.J., 2007. Protected areas and equity concerns. Bioscience 57, 216-221.

Bollersley, T., 1986. Generalized autoregressive conditional heterskedasticity. Journal of Econometrics 31, 307-327.

C.E.Q., 2010. Final recommendations of the interagency ocean policy task force, p. 77.

Chandra, S., Shadel, W.G., 2006. Crossing disciplinary boundaries: applying financial portfolio theory to model the organization of the self-concept. Journal of Research in Personality 41, 346-373.

Crowe, K.A., Parker, W.H., 2008. Using portfolio theory to guide reforestation and restoration under climate change scenarios. Climatic Change 89, 355-370.

Edwards, S.F., Link, J.S., Rountree, B.P., 2004. Portfolio management of wild fish stocks. Ecological Economics 49, 317-329.

Ehrgott, M., 2005. Multicriteria Optimization, second ed. Springer, New York.
Engle, R.F., 1982. Autoregressive conditional heteroscedasticity with estimates of the variance and United Kingdom inflation. Econometrica 50, 987-1007.

Evans, G.W., Kantrowitz, E., 2002. Socioeconomic status and health: the potential role of environmental risk exposure. Annual Review of Public Health 23, 303331.

Figge, F., 2004. Bio-folio: applying portfolio theory to biodiversity. Biodiversity and Conservation $13,827-849$.

Gatto, M., de Leo, G.A., 2000. Pricing biodiversity and ecosystem services: the neverending story. Bioscience 50, 347-355.

Halpern, B.S., McLeod, K.L., Rosenberg, A.A., Crowder, L.B., 2008. Managing for cumulative impacts in ecosystem-based management through ocean zoning. Ocean \& Coastal Management 51, 203-211.

Huberman, B.A., Lukose, R.M., Hogg, T., 1997. An economics approach to hard computational problems. Science 275, 51-54.

Jones, P.J.S., 2009. Equity, justice and power issues raised by no-take marine protected area proposals. Marine Policy 33, 759-765.

Knoke, T., 2008. Mixed forests and finance - methodological approaches. Ecological Economics 65, 590-601.

Koellner, T., Schmitz, O.J., 2006. Biodiversity, ecosystem function, and investment risk. Bioscience 56, 977-985.

Leeworthy, V.R., Wiley, P.C., Stone, E.A., 2005. Socioeconomic Impact Analysis of Marine Reserve Alternatives for the Channel Islands National Marine Sanctuary, ed. U.S.D.O. Commerce. National Oceanic and Atmospheric Administration, Silver Spring, MD.

Markowitz, H., 1952. Portfolio selection. The Journal of Finance 7, 77-91.

Markowitz, H., 1959. Portfolio Selection: Efficient Diversification of Investments. John Wiley \& Sons, New York.

Mascia, M.B., Claus, C.A., 2009. A property rights approach to understanding human displacement from protected areas: the case of marine protected areas. Conservation Biology 23, 16-23.

McClanahan, T.R., Cinner, J., Kamukuru, A.T., Abunge, C., Ndagala, J., 2008. Management preferences, perceived benefits and conflicts among resource users and managers in the Mafia Island Marine Park, Tanzania. Environmental Conservation 35, 340-350.

Mutz, K., Bryner, G., Kenney, D., 2002. Justice and Natural Resources: Concepts, Strategies, and Applications. Island Press, Washington, DC.

OC, P., 2003. America's Living Oceans: Charting a Course for Sea Change. Pew Oceans Commission, Arlington, VA

Sanchirico, J.N., Smith, M.D., Lipton, D.W., 2008. An empirical approach to ecosystem-based fishery management. Ecological Economics 64, 586-596.

Schindler, D.E., Hilborn, R., Chasco, B., Boatright, C.P., Quinn, T.P., Rogers, L.A., Webster, M.S., 2010. Population diversity and the portfolio effect in an exploited species. Nature 465, 609-613.

Sharpe, W.F., 1966. Mutual fund performance. Journal of Business 39, 119-138.

Sutinen, J.G., Kuperan, K., 1999. A socioeconomic theory of regulatory compliance. International Journal of Socio Economics 26, 174-193.

Tilman, D., Reich, P.B., Knops, J.M.H., 2006. Biodiversity and ecosystem stability in a decade-long grassland experiment. Nature 441, 629-632.

USCOP, 2004. An Ocean Blueprint for the 21st Century: Final Report of the US Commission on Ocean Policy. US Commission on Ocean Policy [USCOP], Washington, DC.

Varian, H.R., 2005. Intermediate Microeconomics: A Modern Approach, seventh ed. W.W. Norton. 